\title{
Precision Near-Infrared Photometry for Exoplanet Transit Observations. I. Ensemble Spot Photometry for an All-Sky Survey
}

\author{
C. Clanton ${ }^{1,2}$ C. Beichman, ${ }^{3,4}$ G. Vasisht, ${ }^{3}$ R. Smith, ${ }^{1}$ And B. S. Gaudi ${ }^{2}$ \\ Received 2012 February 20; accepted 2012 May 25; published 2012 June 25
}

\begin{abstract}
Near-IR observations are important for the detection and characterization of exoplanets using the transit technique, either in surveys of large numbers of stars or for follow-up spectroscopic observations of individual planets. In a controlled laboratory experiment, we imaged $\sim 10^{4}$ critically sampled spots onto an Teledyne Hawaii-2RG (H2RG) detector to emulate an idealized star field. We obtained time-series photometry of up to $\simeq 24 \mathrm{hr}$ duration for ensembles of $\sim 10^{3}$ pseudostars. After rejecting correlated temporal noise caused by various disturbances, we measured a photometric performance of less than $50 \mathrm{ppm} \mathrm{hr}^{-1 / 2}$ limited only by the incident photon rate. After several hours we achieved a photon-noise-limited precision level of 10-20 ppm after averaging many independent measurements. We conclude that IR detectors such as the H2RG can make the precision measurements needed to detect the transits of terrestrial planets or to detect faint atomic or molecular spectral features in the atmospheres of transiting extrasolar planets.
\end{abstract}

Online material: color figures

\section{INTRODUCTION}

The dramatic results of the Kepler and COROT missions (Borucki \& Koch 2011; Baglin et al. 2009), as well as earlier ground-based surveys, have demonstrated the power of the transit method for discovering and characterizing other planetary systems. The combination of a planetary radius from a transit measurement with a mass from a radial velocity measurement yields a planet's density and, thus, clues to its composition and evolutionary history. Optical and infrared observations can determine a planet's albedo and equilibrium temperature and even probe global atmospheric circulation patterns. Spectroscopic transit observations have identified atomic and molecular species, including $\mathrm{H}_{2} \mathrm{O}, \mathrm{CO}_{2}$, and $\mathrm{CH}_{4}$ in the atmospheres of planets orbiting nearby bright stars.

The critical instrumental requirement for all these observations is highly precise differential photometry, since the transit of a Jupiter in front of a solar-type star $\left(1 R_{\odot}\right)$ causes only a $1 \%$ decrease in brightness, while the transit of an Earth in front of the same star causes only a $0.0084 \%$ dip (where $0.01 \%=$ $100 \mathrm{ppm}$ ). Wide-field surveys from the ground are typically limited in precision by residual atmospheric effects to 2000 4000 ppm: e.g., Super-WASP (Pollacco et al. 2006) and

\footnotetext{
${ }^{1}$ Caltech Optical Observatories, California Institute of Technology, Pasadena, CA 91125; chas@pop.jpl.nasa.gov,rsmith@astro.caltech.edu.

${ }^{2}$ Department of Astronomy, Ohio State University, Columbus, OH 43210.

${ }^{3}$ Jet Propulsion Laboratory, California Institute of Technology, 4800 Oak Grove Drive, Pasadena, CA 91107; gv@jpl.nasa.gov.

${ }^{4}$ NASA Exoplanet Science Institute, California Institute of Technology, 770 South Wilson Avenue, Pasadena, CA 91125.
}

HATNet (Bakos et al. 2009). Higher-precision measurements $(<1000 \mathrm{ppm})$ have been demonstrated with concentrated observations of individual stars (Irwin et al. 2010; Johnson et al. 2009). From space, however, much higher levels of precision can be achieved for hundreds of thousands of objects simultaneously. The Kepler and COROT missions have demonstrated photometric precision in the visible using CCD arrays as low as $15 \mathrm{ppm}$, limited in many cases by stellar variability itself (Jenkins et al. 2011b). Spitzer and Hubble Space Telescope (HST) photometry and spectroscopy using earlier generations of near- and mid-IR detectors have demonstrated precision of around 100 ppm (Knutson et al. 2007; Swain et al. 2009; Deming et al. 2006).

There are many advantages to carrying out transit observations in the near-IR compared with the visible band: the photon fluxes from $\mathrm{K}$ and $\mathrm{M}$ stars are higher in the near-IR, up to a factor of 50-100 for the coolest $\mathrm{M}$ stars; total photon fluxes from hotter $\mathrm{F}$ and $\mathrm{G}$ stars are a factor of $\sim 2$ higher in a wavelength band that extends out to $1.6 \mu \mathrm{m}$, rather than in a visible band that cuts off at the $1 \mu \mathrm{m}$ CCD limit; starspot noise is reduced compared with visible wavelengths, due to the lower contrast between areas with typical spot and photospheric temperatures (Frasca et al. 2009); and the effects of limb-darkening are greatly reduced, simplifying extraction of planetary parameters from the observations (Désert et al. 2011).

The detection of transiting $\mathrm{K}$ and $\mathrm{M}$ stars is of great scientific interest, since the smaller radii of late-type stars enhances the signals from Earth-sized planets (Gould et al. 2003). The transit of an Earth in front of an M8 star produces a 1\% brightness dip, 100 times larger than for a G2 star. Additionally, the location of 


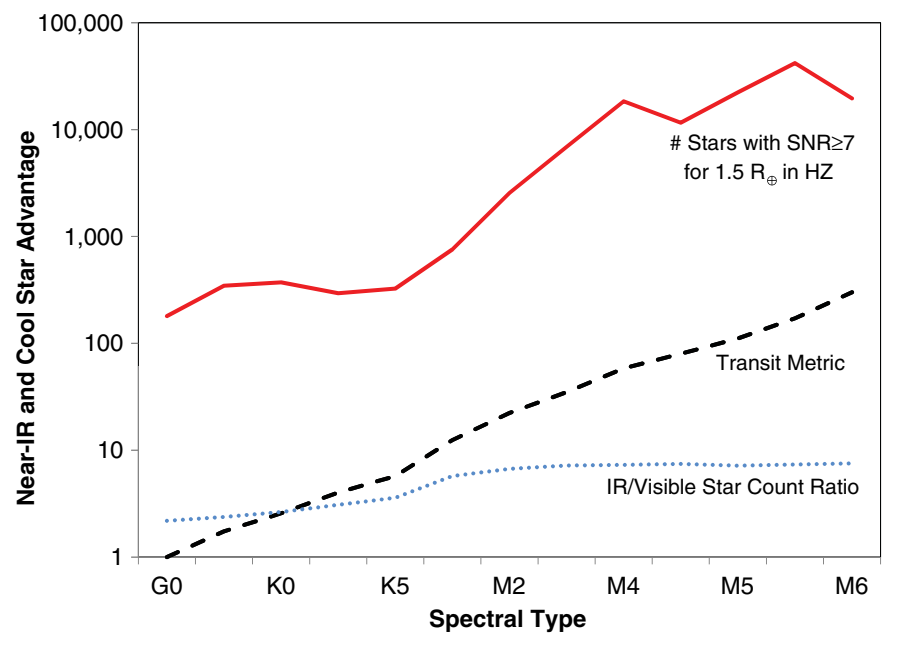

Fig. 1.-The 10- to 1000-fold cool-star advantage of lower-luminosity K and M stars relative to a G0 star for detecting $\mathrm{HZ}$ transits is illustrated by a geometrical metric that takes into account the increased alignment probability, the increased frequency of transits, and the increased transit depth as a function of spectral type (dashed line; Gould et al. 2003). The near-IR advantage (dotted line) is the ratio of the number of stars detectable at a given $\mathrm{S} / \mathrm{N}$ in a broad $J_{W}$ passband $(0.66-1.70 \mu \mathrm{m})$ with H2RG detectors, compared with the number detectable with CCDs working in the visible $(0.6-1.0 \mu \mathrm{m})$. A near-IR survey could detect twice as many planets orbiting F and G stars and between 3 and 7 times more planets around K and M stars. Finally, a Monte Carlo simulation discussed in $\S 5$ shows that $~ 115,000$ stars in an all-sky survey lasting $1.5 \mathrm{yr}$ would be bright enough for the detection of a $1.5 R_{\oplus}$ planet located in the $\mathrm{HZ}$ of its host star ( solid line). In this context, a detection requires the observation of at least three transits and a mission-total $\mathrm{S} / \mathrm{N} \geq 7$. See the electronic edition of the PASP for a color version of this figure.

the habitable zone (HZ), where a planet's temperature might allow for the existence of liquid water, moves in closer to the star, $a_{\mathrm{HZ}} \propto L_{\star}^{1 / 2} \propto M_{\star}^{\alpha / 2}$ with $\alpha \approx 3.4$, increasing both the probability of a geometrical alignment and the frequency of transits (a few days or weeks, compared with a year for a G2 star). Initial results from the Kepler mission suggest that lowmass stars have an increased incidence of low-mass planets (Howard et al. 2011), further enhancing the importance of later spectral types. Figure 1 highlights the increased detectability of transiting planets in the habitable zones of late-type stars. The recognition that cool stars offer an excellent opportunity for detecting $\mathrm{HZ}$ planets has led to ground-based searches directed at M dwarfs (Charbonneau et al. 2009) and has generated considerable interest in improving near-IR Doppler precision (Reiners et al. 2010; Bean et al. 2010; Plavchan et al. 2011).

The large signal from a planet transiting a cool star also enhances the spectroscopic potential of these systems. As pointed out by Deming et al. (2009), transit observations using the James Webb Space Telescope (JWST) could identify numerous atomic and molecular species in the atmosphere of a $\sim 2 R_{\oplus}$ planet transiting a M5 star. Future missions have also been proposed to exploit the potential of spectroscopy to characterize exoplanet atmospheres. A critical need for successful transit spectroscopy is planets orbiting nearby bright stars so that the necessary precision can be achieved in a reasonable time. Kepler and COROT targets are too faint for this purpose, and planets discovered by ground-based transit surveys are too few in number and limited to relatively large radii, due to the constraints of observing through the atmosphere.

Because nearby bright stars are distributed isotropically, there is interest in conducting an extremely wide field-of-view panoramic survey using a low-cost orbital platform (Ricker et al. 2010; Deming et al. 2009; Beichman et al. 2009). Such a survey would target 1-2 million stars, which would be at least $5 \mathrm{mag}$ brighter than the Kepler stars. Such a survey could be conducted with multiple $12.5 \mathrm{~cm}$ apertures, a factor of 10 smaller than Kepler's $1 \mathrm{~m}$ telescope, operating either in the visible using CCD focal planes (Ricker et al. 2010; Deming et al. 2009; Claudi 2010) or in the near-infrared (Beichman et al. 2009). Shifting the observational band from the visible $(0.6-1.0 \mu \mathrm{m})$ into the near-IR $(0.66-1.65 \mu \mathrm{m})^{5}$ for otherwise-identical survey facilities results in a 3- to 10-fold increase in the numbers of cool stars (K5-M5) bright enough for the detection of $\sim 1 R_{E}$ planets (dashed line in Fig. 1). A Monte Carlo simulation (§ 5) shows that an all-sky survey of 2 million stars would include $\sim 150,000$ stars bright enough to allow the detection of planets as small as $1.5 R_{\oplus}$ located in the $\mathrm{HZ}$ of its host star with a mission-total signal-to-noise ratio $(\mathrm{S} / \mathrm{N})$ of 7 or higher. The vast majority of these stars have spectral types later than $\mathrm{M} 0$ and are thus preferentially detected in the near-IR.

The increased near-IR yield over the visible comes from the increased $\mathrm{S} / \mathrm{N}$ in the near-IR, due to stronger photospheric emission in the near-IR (Hauschildt et al. 1999) and/or broader measurement bandwidth. The star counts above a given flux threshold increase as the photon luminosity in a bandpass $\left(L_{\lambda} \Delta \lambda\right)$ to the $3 / 2$ power. The number of stars above a given S/N threshold goes as $N \propto \mathrm{S} / \mathrm{N}^{-3}\left(L_{\lambda} \Delta \lambda\right)^{3 / 2}$; thus, by increasing $L_{\lambda} \Delta \lambda$, the number of stars above a fixed $\mathrm{S} / \mathrm{N}$ increases as $\left(L_{\lambda} \Delta \lambda\right)^{3 / 2}$. Figure 1 also shows that the near-IR measurements do as well as, or better than, a comparable visible-light mission for hotter F, G, and $\mathrm{K}$ stars for the reasons mentioned above.

It is with these scientific goals in mind-an all-sky survey to identify transiting planets with an emphasis on cool $\mathrm{K}$ and $\mathrm{M}$ stars and follow-up spectroscopic observations using stable space telescopes - that we have initiated a series of laboratory experiments designed to probe the ultimate limits of precision photometry using near-IR detectors. We report on the photometric properties of Teledyne's widely used infrared H2RG detector, ${ }^{6}$ which offers near-unity quantum efficiency in the near-infrared (Beletic et al. 2008). In this article we describe laboratory experiments $(\S 2)$ to demonstrate the technical readiness of space-borne transit experiments using existing near-IR

\footnotetext{
${ }^{5}$ The short-wavelength cutoff is selected to exclude $\mathrm{H} \alpha$ emission, which can be variable in cool stars. The long-wavelength cutoff is tailored to exclude small temporal or spatial variations in detector $\mathrm{QE}$ at the limit of its responsivity.

${ }^{6} \mathrm{H} 2 \mathrm{RG}$ or HAWAII-2RG: HgCdTe Astronomical Wide Area Infrared Imager.
} 
detectors. In a future article we will describe the results of simulated differential spectroscopy planned for the JWST and proposed for other missions.

\subsection{Photometry with Infrared Focal Planes}

There is broad interest in the performance characteristics of the next generation of IR detectors. H2RGs will be flown on multiple JWST instruments (Clampin 2010; Deming et al. 2009) and may also form the basis for dedicated exoplanet observatories such as the candidate explorer mission FINESSE and the proposed European Exoplanet Characterization Observatory (ECHO; Tinetti et al. 2011; Tessenyi et al. 2012). Although various investigators have measured relevant device-level properties such as the subpixel nonuniformity (Barron et al. 2007; Hardy et al. 2008), the count-rate-dependent nonlinearity or reciprocity failure (Biesiadzinski et al. 2011), and image persistence (Smith et al. 2008), there has not been a systematic study of the limits to high-precision time-series measurements.

Our goal is to assess the precision possible with the current generation of NIR devices and whether it is possible to achieve IR photometry that is significantly better than what is being achieved from space today. The current state of the art is defined by Spitzer and the Hubble Space Telescope. Observations to determine atmospheric composition by detecting variations in the apparent size of the planet as a function of wavelength using filter photometry or low-dispersion spectra have had notable successes, but also some controversy. Claims have been made about detection of water in Spitzer transit and secondary-eclipse photometry (Barman 2007, 2008; Tinetti et al. 2007), as well as counterclaims (Désert et al. 2009)—sometimes using the same data set. Similarly, the detection of molecular features due to methane, water, and carbon dioxide in NICMOS spectrophotometry is controversial (Swain et al. 2009; Gibson et al. 2011). Observations of the hot Neptune GJ 436b have also led to controversial claims regarding the presence or absence of methane in the atmosphere (Stevenson et al. 2010; Beaulieu et al. 2011; Knutson et al. 2011). To a great extent, it is the treatment of small, systematic detector effects that lie at the heart of these debates.

The results to date suggest that the instrumental precision of Spitzer IRAC photometry and HST NICMOS spectroscopy is $\sim 100$ ppm, although recent improvements in Spitzer's pointing stability have improved IRAC's photometric precision to less than $50 \mathrm{ppm}$ (Demory et al. 2012). A combination of factors, including detector nonlinearities, poor sampling of the pointspread function (PSF), short-term wobbles, and long-term drifts in telescope pointing change the measured signal from the star by several percent over the course of an hour and make the extraction of millimagnitude features from IRAC light curves very challenging. Similarly, Hubble's low Earth orbit introduces systematic effects on the transit timescale (Swain et al. 2008, 2009; Gibson et al. 2011; Berta et al. 2011).

\subsection{Light-Curve Measurements}

When measuring transit light curves, the timescale of relevance is of the order of the transit duration, $t_{d}$. An EarthSun transit produces a characteristic signal of depth of $84\left[\left(R_{p} / R_{\oplus}\right)\left(R_{\odot} / R_{*}\right)\right]^{2} \mathrm{ppm}$, with a maximum duration of $13 \mathrm{hr}$. More generally, the maximum duration with a star-planet separation of $a$ (in $\mathrm{AU})$ is $t_{d} \simeq 13(a / \mathrm{AU})^{1 / 2}\left(M_{*} / M_{\odot}\right)^{1 / 2} \mathrm{hr}$, assuming $R_{*} \propto M_{*}$, which is a good approximation for the lower main sequence. For a solar-type star, transits by planets with separations of a $\sim 0.01,0.1$, and $1 \mathrm{AU}$ have durations of $\sim 1,4$, and $13 \mathrm{hr}$, respectively. Three distinct sources of noise establish the detection threshold of a transit observation over the measurement time $t$ : (1) noise from photon arrival statistics with a standard deviation $\sigma_{N}$; (2) noise from intrinsic stellar variability, $\sigma_{S}$; and (3) instrument measurement noise, $\sigma_{I}$. The total photometric noise $\sigma_{T}$ is given by

$$
\sigma_{T}=\left(\sigma_{N}^{2}+\sigma_{S}^{2}+\sigma_{I}^{2}\right)^{1 / 2} .
$$

In Kepler parlance, $\sigma_{T}$ is called the combined differential photometric precision (Jenkins et al. 2011b).

A few general remarks are important to consider when designing a laboratory experiment to test the limits of detector performance in this application. First, the photometric noise $\sigma_{T}$ is inherently a differential measurement, because only brightness differences in and out of transit are important. Thus, neither absolute photometric calibration nor instrumental or stellar variability changes with timescales much longer than a few times $t_{d}$ are relevant to the measurement. Second, over the long term (i.e., several transits), the stellar noise, $\sigma_{S}$, should average down to $10 \mathrm{ppm}$ for quiescent stars (Basri et al. 2010; Ciardi et al. 2011). The Sun and some of its G dwarf cousins are relatively quiet in this respect (Basri et al. 2010; Jenkins et al. 2011a), with $10^{3} \mathrm{ppm}$ variation on timescales of a rotation period or longer, but with relatively little variability on the 10-13 hr timescale of an Earth transit. While some stars are as quiet as the Sun (10 ppm) on timescales of a few hours, Kepler has shown that at visible wavelengths the average solartype star is somewhat noisier than the Sun (10-30 ppm; Jenkins et al. 2011b). The vast majority of field M dwarfs observed by Kepler have short-term variability $(0.5$ to $12 \mathrm{hr}$ ) less than $1000 \mathrm{ppm}$ and many with less than $100 \mathrm{ppm}$ (Ciardi et al. 2011; Bryden et al. 2010). However, it is important to note that variability due to starspots will be a factor of 2-3 smaller in the near-IR than in the visible, due to reduced contrast in the Rayleigh-Jeans portion of the spectrum (Frasca et al. 2009). Finally, in a properly designed system, $\sigma_{I} \simeq \sigma_{S}$, so that little is to be gained by reducing any one of the noise components to be significantly smaller than the total. The goal of our experiments is to identify methods of reducing $\sigma_{I}$ so that it is comparable with the other irreducible terms (10-20 ppm). Quantifying the magnitude of these effects and demonstrating an ability to reject them as part of a total error budget is critical to designing a particular experiment. Major contributions to $\sigma_{I}$ in transit experiments are 
pixel-scale detector inhomogeneities (flat-fielding errors) convolved with pointing changes, responsivity drifts with temperature and other environmental effects, and detector nonlinearities such as latent image generation.

In $\S 2$, we describe our experiments and data acquisition. In $\S 3$, we discuss our methods and describe the custom dataanalysis pipeline that was written to analyze the large quantities of data that were produced by the experiments (up to 200 Gbytes day $^{-1}$ ). Results are presented in $\S 4$.

\section{DESCRIPTION OF THE EXPERIMENT}

The experimental setup described in this section was used to investigate three major sources of degradation in high-precision differential photometry: (1) long-term ( $24 \mathrm{hr})$ drifts, including the response of the detector and warm electronics, variation in brightness of the illuminating LED, and environmental factors such as dewar temperature; (2) effects of simulated pointing drifts and offsets; and (3) effects of changes in detector temperature. The magnitude of these effects and the development of mitigation techniques are of direct relevance to future space missions, where the expense of controlling parameters such as detector temperature and spacecraft pointing must be traded against the exacting demands of transit measurements.

A $1.7 \mu$ m cutoff $\mathrm{HgCdTe}$ hybrid CMOS detector with $2044 \times$ 2044 imaging pixels on $18 \mu \mathrm{m}$ pitch, Teledyne H2RG-103, was mounted on a temperature-controlled molybdenum plate inside a sealed chamber refrigerated to $77 \mathrm{~K}$ by a liquid-nitrogen dewar with a greater than $20 \mathrm{hr}$ hold time. The wavelength dependence

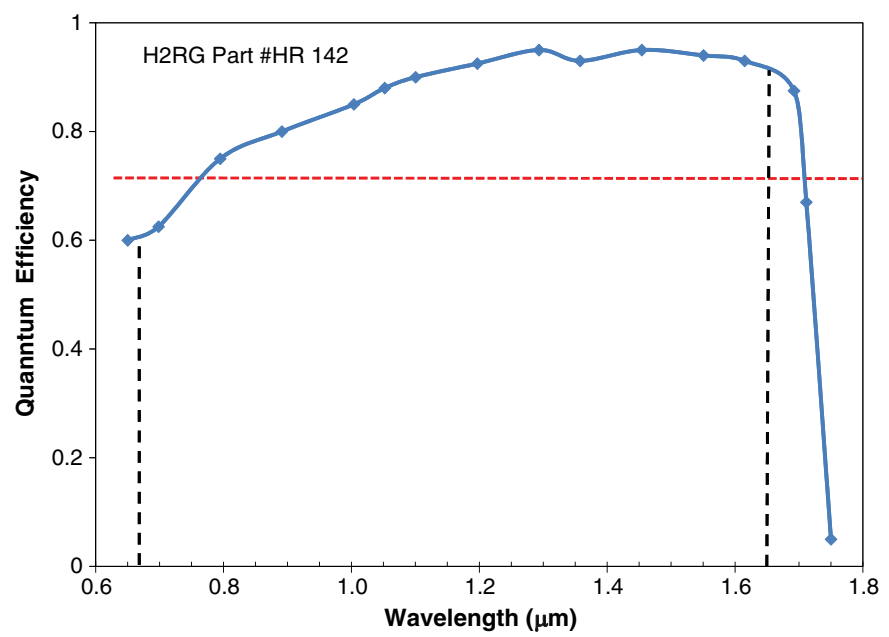

FIG. 2.-Substrate-removed H2RG (Beletic et al. 2008) detector used in these experiments shows high QE ( $>85 \%$ ) over a broad visible and near-IR band out to $1.7 \mu \mathrm{m}$ at an operating temperature of $145 \mathrm{~K}$. The horizontal dashed line shows a typical mission requirement that should be readily achievable with existing technology. Vertical dashed lines show a nominal system passband designed to limit variability due to stellar $\mathrm{H} \alpha$ emission at the short-wavelength end $(\lambda>0.66 \mu \mathrm{m})$ and temperature-induced detector $\mathrm{QE}$ variations at the longwavelength end $(\lambda<1.65 \mu \mathrm{m})$. See the electronic edition of the PASP for a color version of this figure. of the detector responsivity is shown in Figure 2. The dewar was filled $\sim 1 \mathrm{hr}$ prior to each experiment to allow for thermal stabilization and thereafter was automatically refilled every $20 \mathrm{hr}$ from a 160 liter storage dewar so that the detector never warmed up.

The dark chamber was evacuated through a $2.5 \mathrm{~mm}$ diameter serpentine black-painted tube to block entry of thermal emission from warmer parts of the dewar. Light leaks and thermal background were undetectable even in $4 \mathrm{hr}$ exposures against a $0.001 e^{-} \mathrm{s}^{-1}$ pixel $^{-1}$ dark current floor obtained by cooling the detector to $110 \mathrm{~K}$. At $140 \mathrm{~K}$, this detector exhibits median dark current less than $0.02 e^{-} \mathrm{s}^{-1}$ pixel $^{-1}$, which is negligible compared with all signals in this experiment. The $\sim 20 e^{-}$pixel $^{-1}$ read noise (for correlated double sampling) from the 150 pixels in the signal and background apertures combined contributes less than one-third of the total variance in the signal, compared with the signal per pseudostar of $300,000 e^{-}$per $1.2 \mathrm{~s}$ integration.

The layout of the dark chamber is shown in Figure 3. Two LEDs within the dark chamber (wavelengths of 0.890 and $1.30 \mu \mathrm{m})$ are driven by precision current sources external to the dewar. They shine concurrently into a $2^{\prime \prime}$ integrating sphere with a finely textured gold interior and a precision $1 \mathrm{~mm}$ exit aperture. The whole illumination system is housed behind a baffle so that only light from the exit port reaches the detector $139 \mathrm{~mm}$ away. A second baffle halfway along the light path blocks rays scattering off the sidewalls and shades the lenslet supports. A $40 \mathrm{~mm}$ by $40 \mathrm{~mm}$ fused-silica lenslet array made by SUSS Microptics SA (Switzerland), with $300 \mu \mathrm{m}$ lenslet pitch and $4.4 \pm 0.4 \mathrm{~mm}$ focal length, was supported $5 \mathrm{~mm}$ from the detector surface to create a slightly defocused image of the $1 \mathrm{~mm}$ aperture per lenslet. The precisely rectilinear grid of spots was found to have a 17.29 pixel pitch, as expected when projection effects are taken into account.

The full width at half-maximum (FWHM) of each spot was typically $2.3-2.5$ pixels, with low-amplitude diffraction spikes

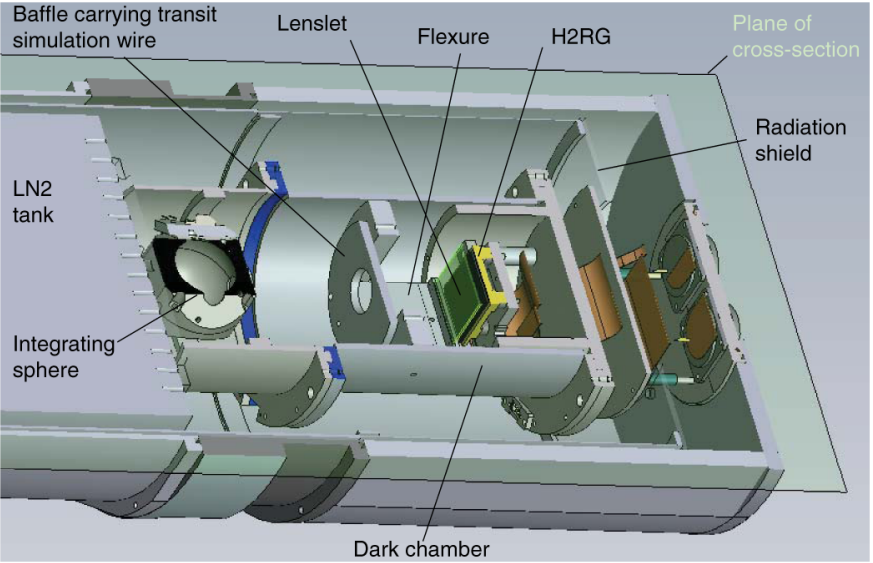

FIG. 3.-Dewar includes an LED illuminating an integrating sphere, a lenslet array, and the H2RG detector. There is no external source of illumination into the dewar. See the electronic edition of the PASP for a color version of this figure. 


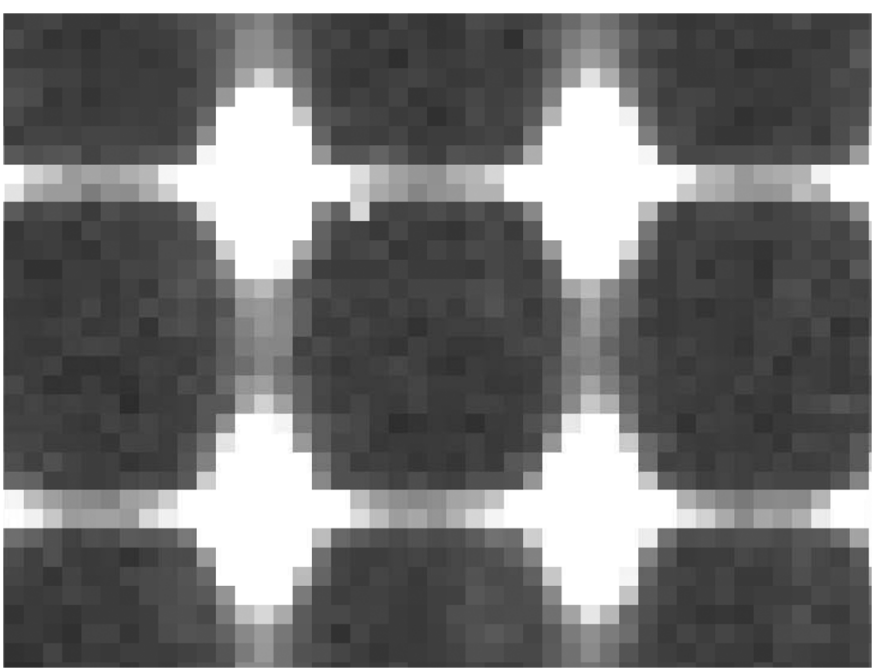

FIG. 4.-Four of the $\sim 10^{4}$ spots with the scale zoomed to show the individual spots plus diffraction spikes.

extending in the vertical and horizontal directions (Figs. 4 and $5)$, as expected for a lenslet array with this pitch. The maximum flux per spot was $\sim 60 \mathrm{ke}^{-} \mathrm{s}^{-1}$, corresponding to the expected count rate from a 9th-magnitude star observed using a camera with a $12.5 \mathrm{~cm}$ aperture in a very broad photometric band extending from 0.66 to $1.65 \mu \mathrm{m}$.

A simple but precise way to move the lenslet array was utilized to emulate small pointing motions. Smooth, reproducible motion with fine resolution was produced by changing the gravity vector acting on the lenslet support formed by two thin cantilevered aluminum sheets, which flex predominantly in one direction. The cylindrical vacuum vessel lies horizontally in a wooden cradle with felt padding, allowing the orientation of the flexures to be changed smoothly with respect to gravity by manually rotating the vacuum vessel about the optical axis.

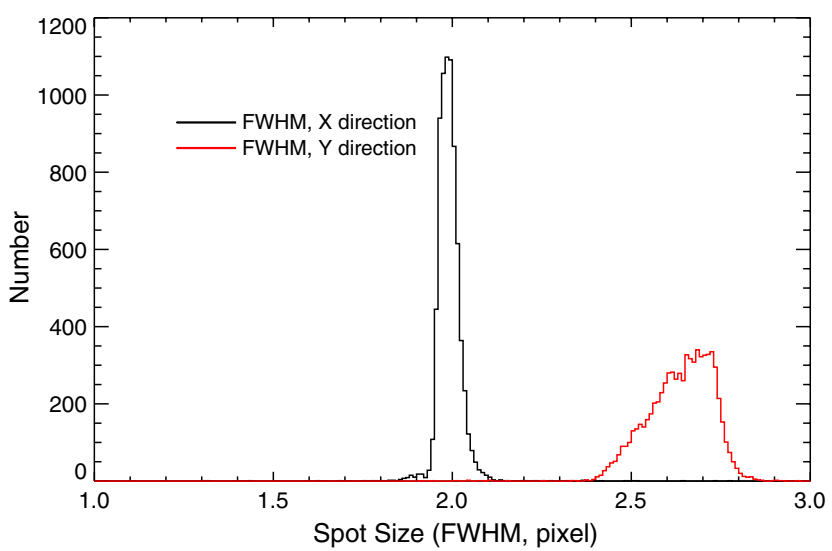

FIG. 5.- Spot images generated by the lenslet array are uniform and slightly elongated. The distribution of spot sizes (FWHM) is narrow and centered around 2.0 pixels in the $X$-direction and 2.5 pixels in the $Y$-direction. See the electronic edition of the PASP for a color version of this figure.
A metal measuring tape attached to the vacuum vessel with a fixed pointer mounted on the cradle serves as an angle indicator. The lenslet position is self-encoding: while the position of any given spot can be can vary due to sampling effects by as much as $180 \mathrm{~nm}$, depending on registration with the pixel grid, this effect averages away rapidly, and averages of 1000 spots exhibit $2 \mathrm{~nm}$ rms temporal stability and comparable coherence under translation when comparing one-half of the spots with the other. Figure 6 shows that the lenslet position varies as the sine of the indicated angle to remarkable accuracy for a hand-driven actuator. Motion up to \pm 8.2 pixels is produced at an angle of $5.2^{\circ}$ from the row direction. The 0.005 pixel $(90 \mathrm{~nm}) \mathrm{rms}$ scatter about a sine fit is dominated by the $0.5^{\circ}$ accuracy with which the dewar rotation angle can be read. This resolution is more than adequate for pointing control, while the $2 \mathrm{~nm}$ temporal stability far exceeds requirements.

Vibration might be a concern with a flexible lenslet support. The resonant frequency of a mass suspended by a spring is $\omega=(g / d)^{1 / 2}$, where $g$ is acceleration due to gravity and $d$ is displacement due to gravity. Measured peak displacement is $148 \mu \mathrm{m}$, so the resonant frequency is expected to be $41 \mathrm{~Hz}$. Indeed, the only resonance detected when applying an impulse to the dewar while reading a small window at $\sim 1200 \mathrm{~Hz}$ was at $41 \mathrm{~Hz}$. No power was detectable at this or any other frequency when the system was not excited. Nonetheless, thick foam padding was placed between the dewar cradle and bench to increase the attenuation 70 -fold at $41 \mathrm{~Hz}$, assuming moderately damped simple harmonic motion.

The detector was read out using a 32-channel Astronomical Research Cameras controller with a $11.44 \mu$ s clock time, including $3 \mu$ s integration time and $1.28 \mu$ s settling time. Correlated double sampling was executed using conventional readout waveforms. As these waveforms were not optimized for

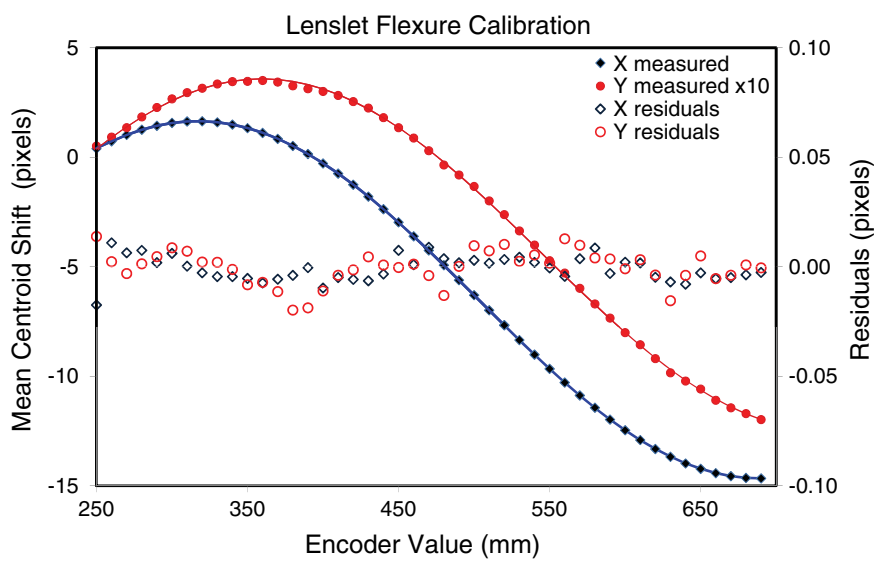

FIG. 6.-As the dewar is rotated around its horizontal axis, the average position of the spots shifts by \pm 8 pixels along the rows ( $X$-direction). A simple sinusoidal model provides a good fit to the data, with residuals at the level of a few hundredths of a pixel. Note that the shifts along columns ( $Y$-direction) are plotted on a 10-times exaggerated scale. See the electronic edition of the $P A S P$ for a color version of this figure. 
such short exposures, there was an exposure duty-cycle penalty, which will be reduced in future experiments. When idle, identical clocking waveforms were executed to maintain a stable self-heating pattern and to avoid abnormal charge accumulation. One full frame scan was assigned to line-by-line reset, rastering through all pixels with identical timing to the readout so that the self-heating footprint during reset matches the read scans. This was immediately followed by a read scan to measure the postreset offset, then by an identical read scan to measure integrated signal plus offset. The difference of the two samples for each pixel is the signal. The exposure time is the delay plus one frame-scan time, while the exposure periodicity is the delay plus three frame-scan times. The exposure duty cycle is their ratio, $33 \%$. To improve the duty cycle, while avoiding some cosmetic defects, the frame-scan time was reduced from $1652 \mathrm{~ms}$ for a full frame to $1221 \mathrm{~ms}$, by skipping 400 lines and then reading a band 1513 lines high.

A typical observing sequence consisted of filling the dewar, setting the LED intensity, and starting a steady cadence of one $1.2 \mathrm{~s}$ exposures every $3.6 \mathrm{~s}$, repeated continuously for periods as long as the $20 \mathrm{hr}$ LN2 autofill interval. This experiment was repeated with the same timing for a range of illumination intensities. In one test, the intensity was deliberately modulated to measure the effectiveness of normalization by the ensemble. In other cases, the dewar was rotated to move the images, simulating pointing changes. Other disturbances such as temperature changes were introduced to assess their importance and our ability to correct for them.

\section{THE ANALYSIS: METHOD AND DATA PIPELINE}

Raw image data for each experiment were run through a data-analysis pipeline that was written specifically for the experimental layout described in $\S 2$. Individual steps of the pipeline are summarized below.

1. Define a region of interest on the array, selecting an area of high cosmetic quality for both the detector and lenslet arrays, roughly 30 by 30 spots in size. For analysis, we typically used 1000 spots, avoiding bad pixels. Channel-to-channel variations in the warm electronics readouts can introduce small measurement perturbations that can be reduced by rigorous design of the warm electronics and standard destriping techniques. Since we wanted to concentrate on the fundamental detector performance issues for these experiments, we avoided pixels spanning channel boundaries.

2. Construct a master dark frame from 200 continuously acquired dark frames and subtract from each image. No flatfield correction was applied to the data, since the analysis steps described below provided the equivalent of a highly localized flat field for each spot.

3. Determine centroids for each star for use as the center for aperture photometry. The IDL routine $\mathrm{FIND}^{7}$ locates sources by fitting a 2D Gaussian, yielding the centroids and FWHM in orthogonal directions for each star. Depending on the experiment, we developed two different ways of placing apertures. In experiments where the stars are stationary with respect to the focal plane, i.e., with no intentional pointing disturbances, we used stationary apertures that remained at a fixed position throughout the photometric sequence. To account for any drifts that may occur, as well as any estimation errors that may arise due to quantization effects of a spot lying on a pixel boundary, the aperture centers are computed as follows: the first few $(\sim 5)$ frames of the sequence are averaged together and then FIND is used to locate the spots in this averaged frame. The same is done with frames from the middle and end of a sequence. This produces centroids for each spot that are then averaged together to get the final value of the aperture center. Typical image motions from the beginning to the end of the photometric sequence are $\sim 0.03$ pixels and $\sim 0.004$ pixels for $X$ and $Y$ centroids, respectively. In experiments with deliberate pointing disturbances, the apertures are allowed to track the star and the moving centroids are recomputed every frame.

4. Calculate fluxes, $l(t)$, using the IDL routine APER with a 3 pixel radius aperture that captures $\sim 95 \%$ of the point-spread function's encircled energy. The signal contained within a background annulus of inner and outer radii of 5 and 8 pixels was subtracted to remove background counts.

We first use aperture photometry of 10 spots to demonstrate that the detector performance is limited by photon shot noise. Measurements at five different brightness levels were fitted to a noise model (eq. [2]) relating the measured noise to the signal intensity and read noise (RN) in $N_{\text {pix }}=150$ pixels (signal aperture plus background annulus):

$$
\operatorname{noise}\left(e^{-}\right)=\sqrt{\operatorname{signal}\left(e^{-}\right)+(\mathrm{RN})^{2} N_{\text {pix }}},
$$

where the noise and signal in data numbers (DNs) were converted to $\mathrm{e}^{-}$using a conversion gain, G. Dark current is negligible at these signal levels. The solid line in Figure 7 shows the noise model with a conversion gain of $G=4.2 \pm 0.2 e^{-} \mathrm{DN}^{-1}$ and $\mathrm{RN}=15 \pm 2 e^{-}$. The conversion gain includes a correction for interpixel capacitance of 0.81 (Brown et al. 2006).

\subsection{Light-Curve Statistics}

We use the Allan variance (Howe et al. 1981) as the main measurement statistic for assessing photometric precision. For a light curve $l(t)$, the Allan variance $\sigma_{l}^{2}$ over a time lag $\tau$ is the second moment of $L^{+}-L^{-}$:

$$
\sigma_{l}^{2}(\tau)=\frac{1}{2}\left\langle\left(L^{+}-L^{-}\right)^{2}\right\rangle \quad \forall \tau \leq \frac{T}{2},
$$

where $L^{+}$and $L^{-}$are integrals (or, equivalently, the integrals are replaced by sums for periodically sampled, discrete light curves) over adjoining time intervals of duration $\tau$.

\footnotetext{
${ }^{7}$ All IDL routines in this article are from the IDL Astronomy User's Library.
} 


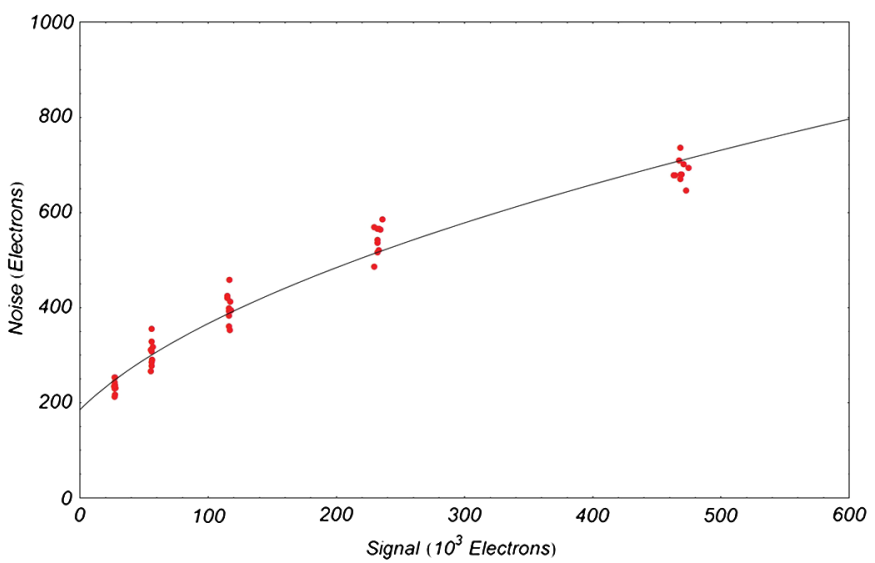

FIG. 7.-Measured noise in 10 photometric apertures is shown as a function of signal brightness along with a simple model combining read noise and photon shot noise (eq. [2]). The measurements are photon shot-noise-limited. See the electronic edition of the PASP for a color version of this figure.

$$
\begin{aligned}
L^{-} & =\frac{1}{\tau} \int_{t-\tau}^{t} l(t) d t, \\
L^{+} & =\frac{1}{\tau} \int_{t}^{t+\tau} l(t) d t,
\end{aligned}
$$

and $T$ is the total duration of the light curve. This statistic preferentially selects noise characteristics on a lag timescale, $\sim \tau$, while rejecting noise on timescales both longer and shorter than $\tau$. For random noise, the variance assumes its usual $1 / \tau$ dependence; in general, $f^{-n}$ noise has $\sigma_{l}^{2} \propto \tau^{n-1}$. It is illustrative to write the Fourier-domain filter form of the Allan deviation as

$$
\sigma_{l}^{2}(\tau)=2 \int_{0}^{\infty} S_{l}(f) \operatorname{sinc}^{2}(\pi \tau f) \sin ^{2}(\pi \tau f) d f
$$

where $S_{l}(f)$ is the power spectrum of $l(t)$. This shows that $S_{l}(f)$ is filtered by a bandpass filter with a response $H_{A}(f)$,

$$
\left|H_{A}(f)\right|^{2}=2 \frac{\sin ^{4}(\pi \tau f)}{(\pi \tau f)^{2}},
$$

peaking at $f \simeq 1.2 / \pi \tau$.

For a given experiment, we calculate an Allan deviation for each star within the detector region of interest. The averaged (or global) Allan deviation is used as the statistic for the determining how well we did over the ensemble of stars in the experi-

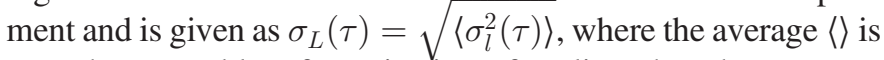
over the ensemble, after rejection of outliers that show exceptionally poor Allan deviations. About $\sim 3-5 \%$ of all stars are rejected as outliers with anomalous Allan deviations. In this article we do not discuss the nature of the outlier stars, other than to say that their spatial distribution is fairly random and that at least some fraction of these stars show poor photometry due to unresponsive pixels located within the aperture.
When no corrections are applied to light curves, $\sigma_{L}(\tau)$ quickly departs from the $\tau^{-1 / 2}$ curve expected for Gaussian noise. The primary culprit at this noise level are the $\sim 10^{3} \mathrm{ppm}$ fluctuations of the lamp on timescales of minutes (Fig. 8). Because the lamp intensity is not explicitly stabilized by feedback, we rely on common-mode rejection of lamp-intensity variations (as described below) to reduce this noise source.

\subsection{Measurement Model and Parameter Decorrelation}

Light curves rapidly depart from the ideal case, due to a number of sources of $1 / f$ noise. Laboratory lamps vary more rapidly than stars, and lamp fluctuations $\left(10^{3} \mathrm{ppm}\right)$ are typically the largest source of nonideal behavior in our experiments. However, the lamp intensity is in common with the spot ensemble, and because we achieve excellent common-mode rejection, lamp variations can be removed to the required accuracy. The stability of the light curves is also affected by environmental factors such as image wander, changes in the optical pointspread function, detector temperature, etc. These changes can be measured and captured in the "instrument state" and then be removed from the data. In general, the light curves $\mathbf{y}$ (vector-valued for $m$ spots) are related to the combined source and instrument state, $\mathbf{x}$, as

$$
\mathbf{y}=\mathbf{F}(\mathbf{x}) .
$$

There are various ways in which to define the vector-valued model function $\mathbf{F}(\mathbf{x})$. The most sophisticated approach is via a full description of the image formation process if one is in possession of the detailed PSF and the detector response function.

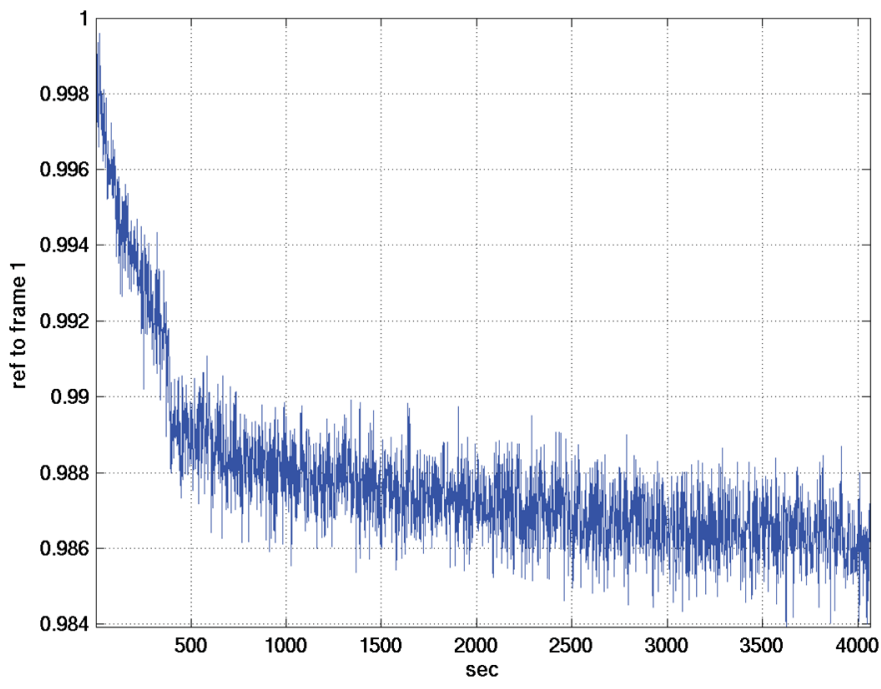

FIG. 8. - Light curve of a typical spot for the first hour after turning on the LED shows a rapid drop in LED brightness of $\sim 1.2 \%$, as expected for diode junction self-heating by $\sim 1 \mathrm{~K}$, followed by a long-term drift of $-0.2 \% \mathrm{hr}^{-1}$. short-term fluctuations at the level of a few parts per thousand are also evident. See the electronic edition of the PASP for a color version of this figure. 
Such an approach may be necessary to obtain good photometric performance in cases when the PSF is poorly sampled and/or if the perturbations are relatively large. In our case, with a wellsampled PSF, and for relatively small perturbations to the instrument state, a simpler parameterized form of $\mathbf{F}(\mathbf{x})$ suffices. We find that in most instances we can linearize equation (3) and rewrite it as

$$
\mathbf{y}=\mathbf{F}\left(\mathbf{x}_{\mathbf{i}}\right)+\mathbf{K}\left(\mathbf{x}-\mathbf{x}_{\mathbf{i}}\right),
$$

where $\mathbf{x}_{i}$ is some initial state, and $\mathbf{K}$ is a $m \times n$ Jacobian matrix with elements given by the partial derivatives

$$
K_{i, j}=\frac{\partial F_{i}(\mathbf{x})}{\partial x_{j}},
$$

relating the photometry for spot $i$ to the instrumental parameter $j$. One of our goals is to quantify the "sensitivities" to various parameters; for any parameter $x_{j}$, this is simply a row average (i.e., equivalent to an average over the spot ensemble) of the Jacobian, $\left\langle K_{i, j}\right\rangle_{i}$.

However, in some cases the truncation to first order (eq. [4]) is insufficient; this is the case if there is relatively large image motion ( $>0.1$ pixel) or if lamp fluctuations are large and pixel response nonlinearities must be considered. If so, $\mathbf{F}$ can be expanded to second order in $\mathbf{x}$. This higher-order model is recommended, for example, for light curves extracted from poorly sampled Spitzer IRAC bands 1 and 2 images (Knutson et al. 2011), requiring estimation of the Hessian:

$$
H_{i, j k}=\frac{\partial F_{i}(\mathbf{x})}{\partial x_{j} \partial x_{k}} .
$$

In our experiments, the state is well determined by the following set of parameters:

1 . The image centroid and pointing drift, $\delta x$ and $\delta y$, are measured in pixels and are usually a small fraction of the pixel pitch. In general, we find that for image motions larger than one-tenth of a pixel, $(\delta x, \delta y \geq 0.1$ pixels), second-order terms in $\delta x$ and $\delta y$ are required to model the photometry. With image motions of $\geq 0.3$ pixels, the second-order model no longer suffices.

2. Changes in the optical point-spread function. Focus drifts can lead to significant changes in the PSF and the photometry. We capture focus with relatively simple parameterization, i.e., we approximate all spots as Gaussians and track the change in the FWHM $\left(\delta w_{x}, \delta w_{y}\right)$ caused by temporal focus drifts. However, we have no way to inject controlled amounts of focus error.

3 . Changes in the lamp intensity $(\delta L)$ are estimated by an average of the light curves of an ensemble of stars randomly distributed over the detector ROI. In typical experiments, about 20 stars are need for a reasonable estimate of $\delta L$.

4. Any changes in the detector temperature $\left(\delta T_{\mathrm{FPA}}\right)$.

Changes in the state parameters are often correlated, being driven by the same underlying mechanism: i.e., the daily thermal cycles between dewar fills. On orbit this could be the solar thermal forcing of a spacecraft. Given these correlations, it is convenient to transform the state $\mathbf{x}$ to $\mathbf{x}^{\prime}$, with the latter having a minimum set of uncorrelated or orthogonal parameters by using a principal components analysis (PCA). PCA is an orthogonal linear transformation that maps a set of observations of possibly correlated vectors into a set of values of orthogonal vectors, called the principal components. By definition the first principal component has the highest variance, i.e., it accounts for as much of the variability in the observed data as possible. Subsequent principal components are ordered by variance, but under the constraint of orthogonality to the preceding components. Our analysis proceeded according to following steps:

1. Let the light curve for each spot consist of $p$ measurements; each measurement yields an estimate of the state parameters. Convert the $p \times 1$ vectors of state parameters $x_{j}$ to centered, rescaled versions via the transformation $\hat{x}_{j}=$ $\left(x_{j}-\bar{x}_{j}\right) / \sigma_{x_{j}}$, where $\bar{x}_{j}$ and $\sigma_{x_{j}}$ are the mean and standard deviation.

2. Construct the $p \times n$ state matrix $\mathbf{X}$ for each spot using a set of $n$ state parameter vectors. Rows of $\mathbf{X}$ correspond to the observations, and columns to the parameters.

3. Use the IDL routine PCOMP $(\mathbf{X})$ to perform principal components analysis on $\mathbf{X}$ and return the principal component coefficients.

4. We generate the new state, $\hat{\mathbf{X}}$, using a truncated set of $m$ principal component vectors ( $m \leq n$, and $m$ is typically 3-4). The normalized light curve $\mathbf{Z}$ is described by $\mathbf{Z}=\hat{\mathbf{X}} \mathbf{K}+\mathbf{e}$, where $\mathbf{e}$ is an error vector. Minimizing the cost, $\mathbf{e}^{\mathbf{T}} \mathbf{e}$, with respect to $\mathbf{K}$, yields a least-squares solution for the sensitivities $\mathbf{K}=\left(\hat{\mathbf{X}}^{\mathbf{T}} \hat{\mathbf{X}}\right)^{-1} \hat{\mathbf{X}}^{\mathbf{T}} \mathbf{Z}$. Repeated tests show the error e to be nearly zero-mean Gaussian, making the least-squares estimate of $\mathbf{K}$ equivalent to a maximum likelihood estimate and conferring upon it a direct probabilistic interpretation.

5. Compute the model light curves, $\hat{\mathbf{X}} \mathbf{K}$. The error residuals, $\mathbf{e}=\mathbf{Z}-\hat{\mathbf{X}} \mathbf{K}$ are used to compute the Allan deviations. Allan deviation curves labeled as "decorrelated" have been corrected for systematics using the above scheme.

\section{RESULTS}

In the simplest experiments, we held the experimental setup constant for approximately $24 \mathrm{hr}$ and either kept the lamp brightness constant or changed the lamp brightness in discrete steps. Figure 9a shows the intensity of a single spot before correction for variations in lamp brightness. The Allan deviation as a function of lag for that single spot (Fig. 9b), inclusive of lamp variations, hits a floor at the $0.1 \%$ (or $1000 \mathrm{ppm}$ ) level. The decorrelation using PCA (with or without clipping of a few bad pixels) greatly reduces the effect of lamp variations and other noise sources, achieving a noise floor of $\sim 50 \mathrm{ppm}$ for a single spot. Data sets from different experimental runs shown 

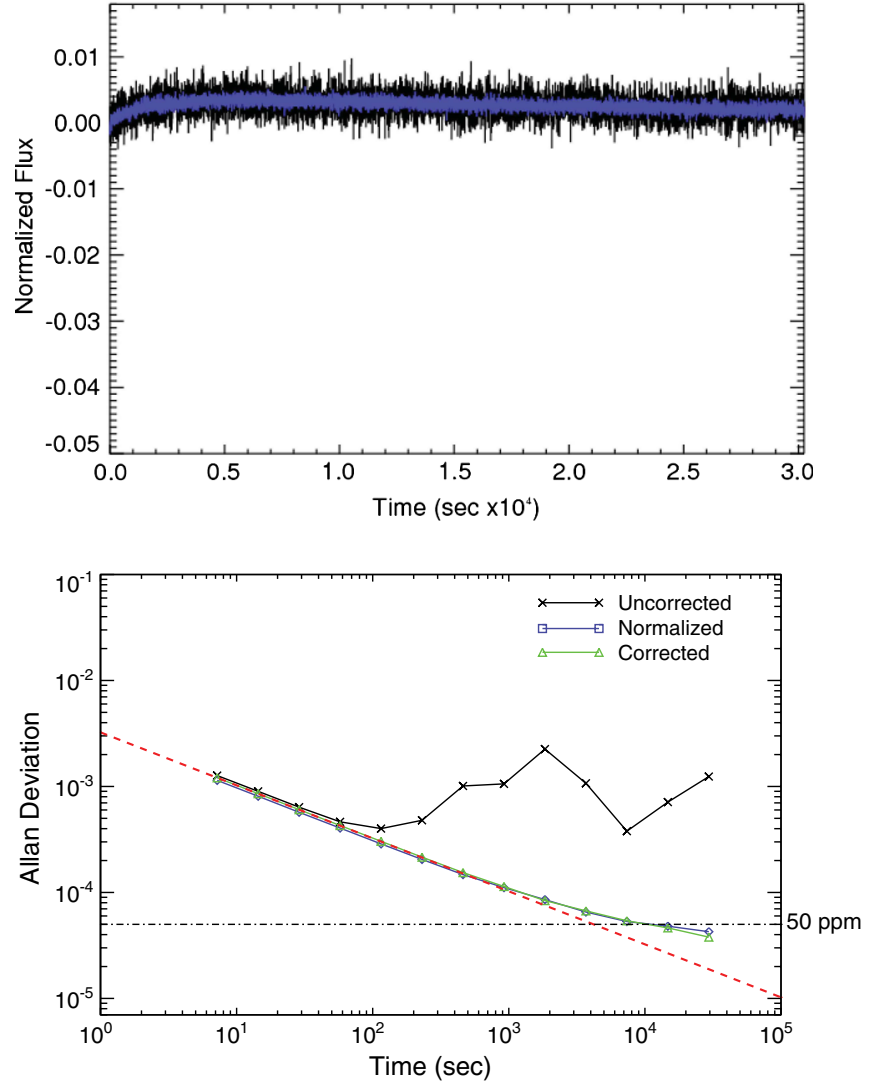

FIG. 9.-Left: A single-spot light curve $\left(I_{i} / \bar{I}-1\right)$ over an $8 \mathrm{hr}$ interval for nominally constant lamp intensity shows variation at the level of a few tenths of a percent (curve). The PCA model is shown as a dashed line. Right: The Allan deviation of the light curve $(x)$, inclusive of lamp variations, hits a floor at the $0.1 \%$ (or $1000 \mathrm{ppm}$ ) level. After PCA decorrelation the noise level obeys a $\tau^{-0.5}$ relationship until reaching a floor of roughly $50 \mathrm{ppm}$ after $4.2 \mathrm{hr}$ for a single spot before (squares) and after (triangles) bad-pixel rejection. See the electronic edition of the PASP for a color version of this figure.

in Figures 10-13 show single-spot noise floors between $~ 30$ and $50 \mathrm{ppm}$.

Figure 10 shows the result of first averaging the light curves of multiple spots together and then computing the Allan deviation of the averaged light curve. At short lag times, the initial noise level decreases as expected for photon-noise-limited measurements: the noise is inversely proportional to the square root of the number of spots, i.e., proportional to the square root of total number of photons. At longer lag times, the limiting noise floor also drops as the square root of number of spots, i.e., as the square root of number of independent, uncorrelated measurements. This result implies that averaging over multiple spots can reduce other temporally correlated noise sources down to the ultimate noise floor seen in these experiments of 10-20 ppm. Figure 11 shows that even large commanded excursions in lamp brightness can be corrected for via ensemble averaging and PCA decorrelation. Overall, these data show that by using decorrelation techniques and suitable averaging of independent

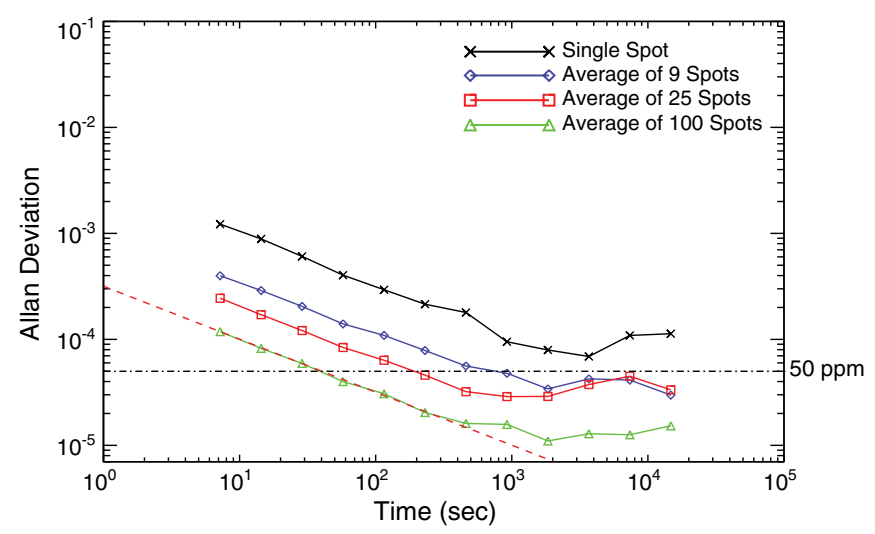

Fig. 10.-Allan deviations of decorrelated light curves, based on data presented in Fig. 9a made by co-adding multiple spots (from top to bottom: $N=1,9,25$, and 100 spots). The noise level decreases as $\sqrt{N}$ at short lags, where the statistics are determined by photon noise. Even at longer lags, where correlated noise dominates single-spot Allan deviations, the improvement is $\sim \sqrt{N}$, suggesting that the systematics are mostly uncorrelated between spots. The dashed line shows that when sufficient flux is included in the measurement, i.e., the 100-spot line, the noise maintains the photon-noise-limited $\tau^{-0.5}$ relationship until reaching a floor of roughly $\sim 10-20 \mathrm{ppm}$. See the electronic edition of the PASP for a color version of this figure.

samples, the H2RG detector can achieve very high precision differential photometry with noise values as low as $\lesssim 20 \mathrm{ppm}$.

\subsection{Pointing Rejection}

In general, the measured count rate for a source represents a convolution of the detector spatial response with the pattern of optical illumination over a predefined aperture. The illumination pattern is the broadband PSF in the case of a survey experiment, or a narrow spectral extraction region in a spectrograph. Because of the convolution the coupling of pointing changes and/or PSF variations with interpixel or intrapixel responsivity variations within the detector changes the measured signal. While a transit survey experiment can correct for gross drifts in detector/electronics response using stellar ensemble averages, changes in the measured brightness of individual stars due to small pointing shifts must be corrected using decorrelation techniques. These techniques have been pioneered using Spitzer and HST data (Knutson et al. 2007; Swain et al. 2008).

The response within a pixel can have substantial structure due to declining detection efficiency at its edges, diffusion of charge into neighboring pixels and electrical crosstalk between pixels. Sensitivity variations across a pixel reduce photometric accuracy in images even where the PSF is fairly well-sampled. The intrapixel variations in the PACE type detectors flown on NICMOS have been found to be quite large (Finger et al. 2000) and clearly affect the light-curve fidelity obtained during NICMOS transit photometry, despite the large (5-pixel FWHM) defocus in the NIC3 camera.

These intra- and interpixel responsivity variations make pointing jitter and wander among the largest terms in the 

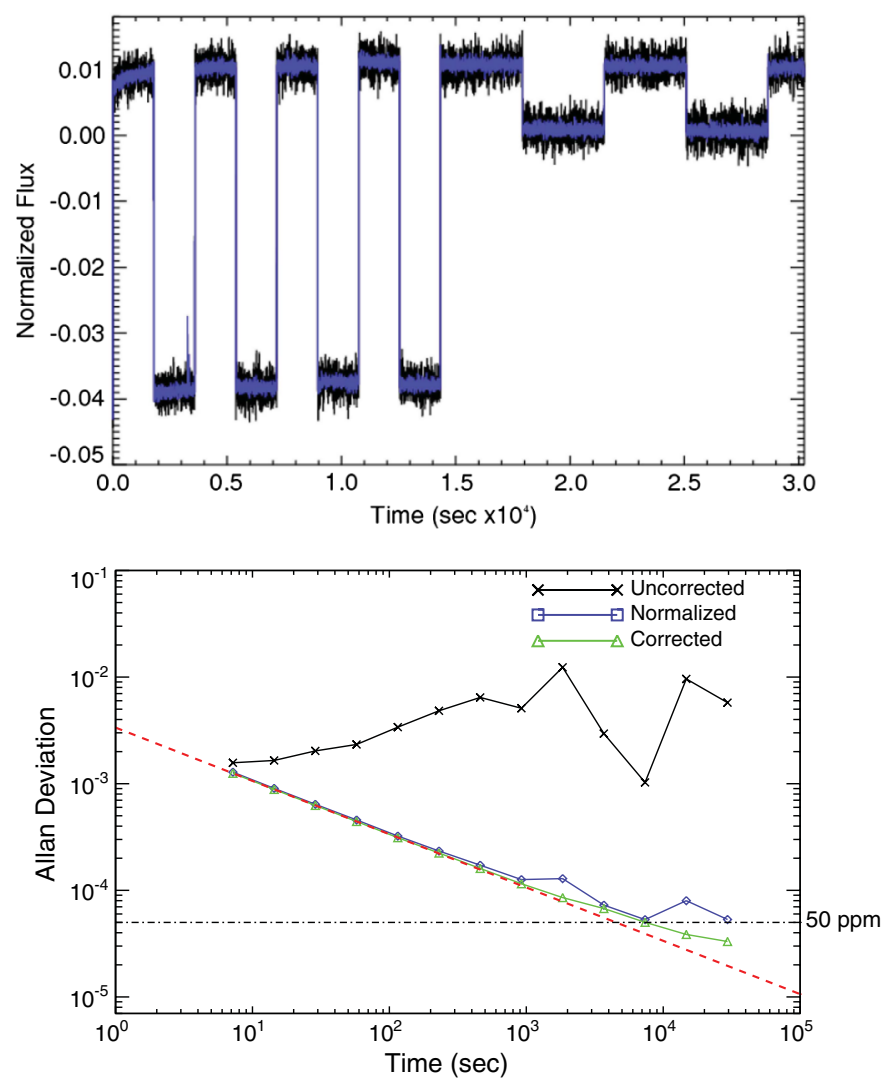

FIG. 11.-Left: The light curve of a single spot following commanded changes in lamp intensity. Right: The averaging characteristics of unnormalized data are in black with $\times$ markers. After normalization by the ensemble average, the noise floor drops to less than $100 \mathrm{ppm}$ (diamonds). After full PCA decorrelation, the noise level follows a $\tau^{-0.5}$ relationship until reaching a floor of roughly less than $50 \mathrm{ppm}$ for a single spot (triangles). The common-mode rejection for lamp variations is better than 100:1. See the electronic edition of the $P A S P$ for a color version of this figure.

photometry error budget in a transit experiments using older detectors. Improvements to quantum efficiency of $\mathrm{HgCdTe}$ detectors in recent years have resulted in most photons being detected if they are not reflected. Even though lateral charge diffusion results in a variation in response of a given pixel, depending on the position of the stellar image, the integrated response remains almost constant. We conducted an experiment to determine the degree to which pointing affects photometric precision, and the degree to which decorrelation analysis can be used to remove these effects from the light curves. Controlled pointing offsets were introduced by rotating the dewar and causing a 1D shift in spot positions (\$2, Fig. 6). A series of dewar rotations produced up to 0.25 pixel steps in the $X$ locations of the spots and much smaller shifts in the $Y$ locations. After determining the new spot centroids, we applied the PCA decorrelation technique and were able to reduce the noise floor to well under $30 \mathrm{ppm}$ as demonstrated in Figure 12. We conclude that in a configuration with $\sim 2$ pixel radius spots, photometry of less than $50 \mathrm{ppm}$ can be achieved using PCA correction, even in the presence of pointing offsets of up to 0.25 pixels.

\subsection{Temperature Dependence}

Thermal fluctuations can produce changes in detector response. Fortunately, the temperature dependence of detector responsivity is relatively weak in modern H2RG arrays compared with variation in the quantum efficiency in the NICMOS 3 devices used on $H S T$. We conducted a simple experiment to determine the degree to which the detector temperature must be controlled to avoid impacting precision photometric requirements. In our setup a temperature controller maintains the focal plane at $140 \mathrm{~K}$ with a precision of $\pm 5 \mathrm{mK}$. An $8.5 \mathrm{hr}$ long photometric image sequence was acquired during which the temperature of the focal plane was modulated by changing
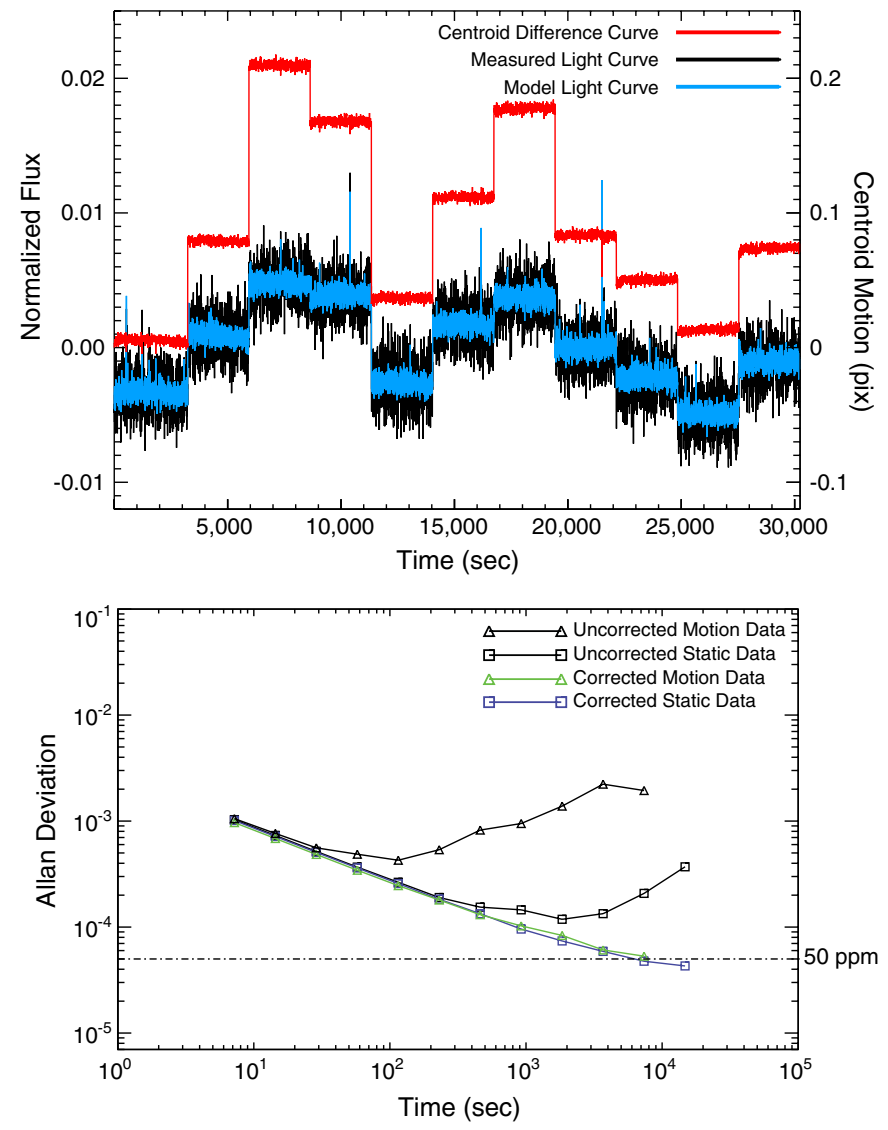

FIG. 12.-Top: Light curve of a single spot in response to commanded image motion. The large steps (right-hand axis in pixel units) represent centroid motions of up to 0.25 pixels. The thick black curve shows the resultant variations in measured signal intensity, up to almost $1 \%$. The lighter-colored (gray) curve shows the PCA model fitted to the light-curve data (bottom). The curves marked with squares denote data with no motion, and the curves with triangles denote data with position offsets. The two lower curves are corrected using PCA analysis. After full PCA decorrelation the noise level follows a $\tau^{-0.5}$ relationship until reaching a floor of roughly $30 \mathrm{ppm}$ for a single spot. See the electronic edition of the PASP for a color version of this figure. 

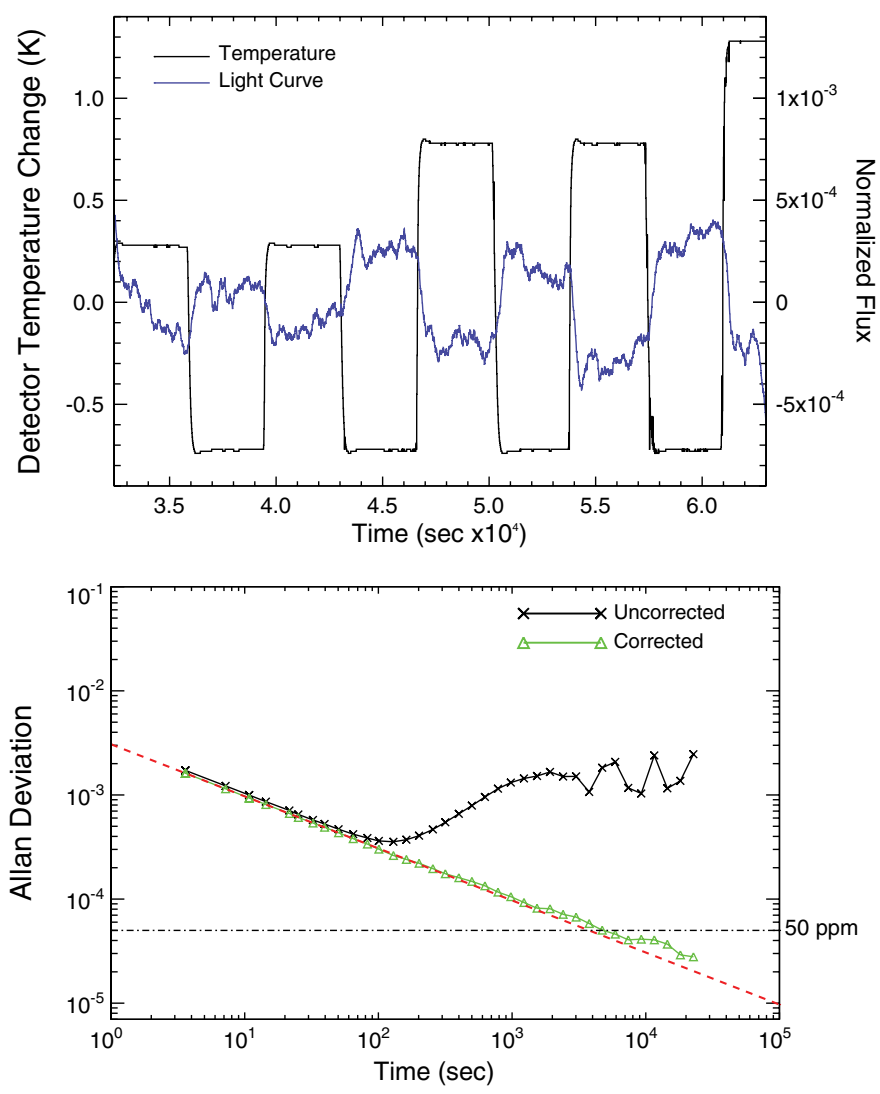

FIG. 13.-Left: A single-spot light curve responding to commanded steps in the detector temperature in black. Right: An Allan deviation plot showing unnormalized data at the few-percent level. After normalization by the ensemble average, the noise floor drops to less than $100 \mathrm{ppm}$. After full PCA decorrelation the noise level follows a $\tau^{-0.5}$ relationship until reaching a floor of roughly less than $30 \mathrm{ppm}$. See the electronic edition of the PASP for a color version of this figure.

the controller set-point temperature by up to $2 \mathrm{~K}$. The temperature steps are shown in Figure 13 along with the change in the average signal. The figure also shows the Allan deviation for the uncorrected and corrected (decorrelated) data. The decorrelation technique can correct for relatively large swings in detector temperature without a significant impact on the final photometric precision.

The average temperature coefficient of the intensity variation found by averaging the light curves of many stars is $\sim 250 \mathrm{ppm} \mathrm{K}^{-1}$. The combination of temperature control at the $0.1 \mathrm{~K}$ level and decorrelation analysis will reduce the effect of temperature fluctuations to acceptable levels. A flight instrument would use a variety of techniques to maintain detector temperature, including heaters at the focal-plane level $(0.05 \mathrm{~K})$, as well as using thin metal traces built into the H2RG multiplexor that can monitor and control the package temperature directly at the few-millikelvin level. Finally, detector reference pixels can be used to suppress offsets due to residual bias voltage and temperature drifts.

\section{DISCUSSION}

\subsection{Applicability to Other Near-IR Focal Planes}

How applicable are these results to Teledyne HxRGs with different cutoff wavelengths? H2RG-103, used in these tests, is from one of the first successful batches of $1.7 \mu \mathrm{m}$ cutoff $\mathrm{HgCdTe}$ detectors made for the SNAP program in 2006. Substrate removal had been mastered, delivering what is believed to be near $100 \%$ internal quantum efficiency: almost all photons passing through the antireflection coating produce signal electrons. A corollary of this high internal QE, shared by devices with other cutoff wavelengths, is that intrapixel response variation is low since there is no charge loss mechanism to modulate sensitivity.

Barron et al. (2007) performed intrapixel response measurements on the near-identical $1.7 \mu$ m cutoff H2RG-102 from the same batch and found that while lateral charge diffusion redistributes charge between neighboring pixels, the integrated flux is independent of the position of subpixel-size spots at the less than $2 \%$ rms level. Hardy et al. (2008) reached the same conclusion after performing similar tests over a wider area on $J W S T$-like H1RGs ${ }^{8}$ (Beletic et al. 2008) with $5 \mu$ m cutoff and found that process-dependent features, such as surface particulates and subpixel width scratches, dominated the response.

The higher bandgap of the $1.7 \mu \mathrm{m}$ detector presents special manufacturing challenges that result in higher read noise and persistence. While operating temperature can be higher for a given dark current, the dark current floor is similar to the longer cutoff devices. For these reasons we believe that the $1.7 \mu \mathrm{m}$ devices represent a worst-case scenario, and that the results in this article are probably extensible to longer cutoff devices. It is often the case that greater differences in performance are observed due to process variations than due to the cutoff itself, but the better devices at any cutoff wavelengths are expected to reach the levels of performance reported here.

Thus, we maintain that our results showing that $1.7 \mu \mathrm{m}$ cutoff H2RG devices are capable of less than $50 \mathrm{ppm}$ precision photometry are directly applicable to longer-wavelength devices such as JWST's $5 \mu \mathrm{m}$ arrays. Our existing experimental setup could be used to probe the behavior of $2-2.5 \mu \mathrm{m}$ cutoff material with only slight modifications. However, operation out to $5 \mu \mathrm{m}$ would require a new experiment designed with much more attention to reduced backgrounds and lower-temperature operation.

\subsection{A Near-IR Transit Survey}

We put this detector performance into context by considering observations with a small near-IR camera $(12.5 \mathrm{~cm}$ diameter) observing in a wide " $J_{W}$ " band extending from 0.66 to $1.65 \mu \mathrm{m}$ where we have taken typical values for the overall optical and

${ }^{8} \mathrm{H} 1 \mathrm{RG}$ or HAWAII-1RG: HgCdTe Astronomical Wide Area Infrared Imager. 
detector quantum efficiency (total of 50\%) and sky brightness in space $\left(0.15 \mathrm{MJy} \mathrm{sr}^{-1}\right)$. In Figure 14 the solid black curve shows the idealized case where the $\mathrm{S} / \mathrm{N}$ varies simply as the square root of the stellar photon flux. Three additional curves show realistic cases taking into account the photon noise from sky background and three different values for the noise floor $(10,50$, and $1000 \mathrm{ppm}$ ). For the bright stars considered here, detector read noise and dark current are negligible sources of noise. For the brightest stars, the $\mathrm{S} / \mathrm{N}$ curves flatten out due to the uncorrected detector responsivity changes, i.e., the flat-field noise floor. At faint magnitudes, photon noise from the sky background reduces the $\mathrm{S} / \mathrm{N}$ relative to the stellar photon-noise limit. The $1000 \mathrm{ppm}$ limit represents the best values that have been obtained in ground-based surveys of very large numbers of stars $(\S 1)$. Horizontal lines denote the $\mathrm{S} / \mathrm{N}$ levels needed to detect the transit of a $1 R_{\oplus}$ planet orbiting $\mathrm{G} 2, \mathrm{~K} 5$, and $\mathrm{M} 3$ stars at the $3 \sigma$ level. The noise floor demonstrated in this article, less than $50 \mathrm{ppm}$, is adequate to detect at Earth transiting a $\mathrm{K}$ star at $10-20 \mathrm{pc}$ in a single $900 \mathrm{~s}$ observation, where $900 \mathrm{~s}$ is a typical observation time for a spacecraft in low Earth orbit. The fact that the noise in the average of independent measurements continues to decrease to $\sim 10-20 \mathrm{ppm}(\S 4)$ implies that weaker transit signals can be extracted from the light curves with suitable processing of multiple transits. Typical requirements for a transit survey demand a minimum of three transits and a total $\mathrm{S} / \mathrm{N}=$ 7 for a confirmed detection.

The yield of a survey depends on many instrumental and survey strategy parameters (e.g., number of cameras, survey

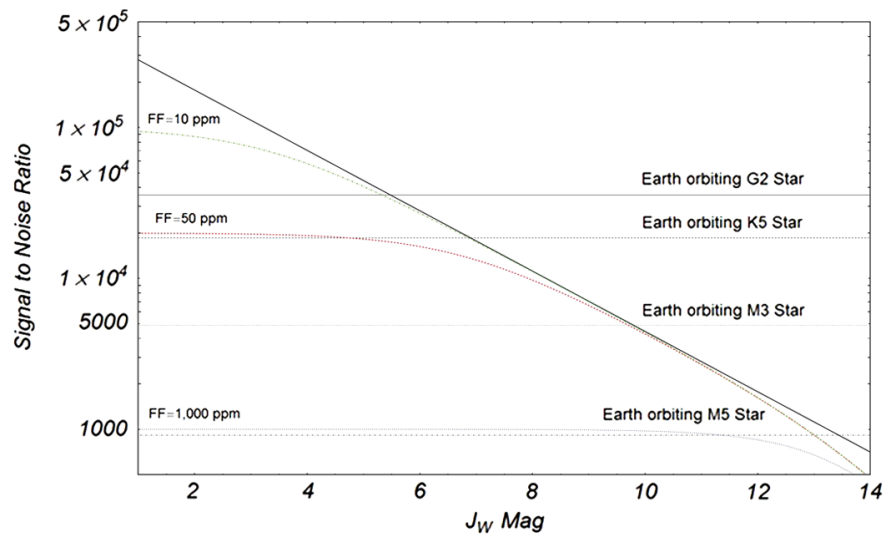

FIG. 14.- S/N for stars as a function of broadband $(0.66-1.70 \mu \mathrm{m}$, denoted $J_{W}$ ) magnitude after a $900 \mathrm{~s}$ integration typical of an observation in low Earth orbit. Observations are assumed to use a camera with $12.5 \mathrm{~cm}$ aperture using a $1.7 \mu \mathrm{m}$ cutoff H2RG detector, as discussed herein. The noise model includes photon noise from the star and the sky, as well as a noise floor set by the limiting detector performance (the flat-field (FF) limit in ppm). The solid black line denotes the noise limit as set only by stellar photon noise. At magnitudes fainter than $J_{W} \sim 14$ mag sky background reduces the $\mathrm{S} / \mathrm{N}$, while for bright stars the residual noise floor dominates the $\mathrm{S} / \mathrm{N}$. The effects of stellar jitter and background confusion are neglected here but are included in the Monte Carlo simulations. The horizontal lines show the $\mathrm{S} / \mathrm{N}$ needed to detect a $1 R_{\oplus}$ planet transiting G2, K5, and M3 stars in a single $900 \mathrm{~s}$ observation at the $3 \sigma$ level. See the electronic edition of the PASP for a color version of this figure. cadence and duration, mission length, etc.) and is beyond the scope of this article. We have developed a Monte Carlo simulation using modest extrapolations of the distributions of the size and orbital location of planets derived from radial velocity and transit surveys (Howard et al. 2011; Borucki et al. 2011). The simulation, which will be described elsewhere, conservatively estimates that for a sample of $2 \times 10^{6}$ stars, each hosting exactly one planet and spanning spectral types from $\mathrm{F} 0-\mathrm{M} 4$ $\left(J_{W}<14 \mathrm{mag}\right)$ and M5-M8 $\left(J_{W}<15 \mathrm{mag}\right)$, a $1.5 \mathrm{yr}$ nearIR survey using six cameras on a spacecraft in a low Earth orbit would detect over $\sim 200$ rocky planets $\left(\lesssim 2 R_{\oplus}\right.$; Fig. 15$)$, of which at least $\sim 50$ would be located in the habitable zones of their $\mathrm{K}$ and $\mathrm{M}$ star hosts. The time any star is monitored varies with ecliptic latitude, $\beta$, and for the particular configuration simulated here we assumed 75 days $\mathrm{yr}^{-1}$ for a star at $\beta=30^{\circ}$. The survey would also identify a few thousand gas-and-ice giant planets covering a broad range of equilibrium temperatures. Some 300 planets would orbit stars brighter than $J_{W}=$ 9 mag and would be suitable for confirmation and mass determination via radial velocity measurements and spectroscopy for the characterization of planetary atmospheres. A survey conducted in a more distant orbit from the Earth, e.g., L2, would

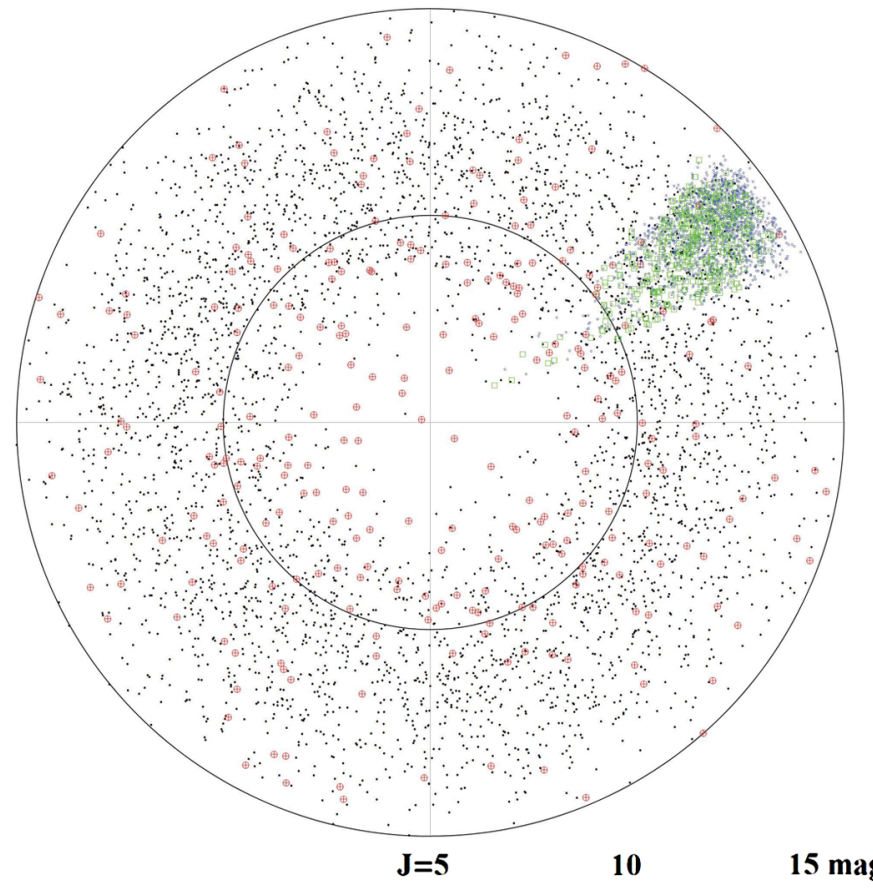

FIG. 15.-Monte Carlo simulation suggests that an all-sky survey would find thousands of gas giant planets and hundreds of rocky planets, many of which would occupy the temperate habitable zones of their late-type host stars. In this polar diagram, the radial coordinate represents a $J$ magnitude and the polar angle represents a simulated sky coordinate. Gas giants from the simulation are shown as small filled circles, and rocky planets are shown as $\oplus$. For comparison, confirmed Kepler planets and Kepler candidates are shown with their $J$ (Two Micron All Sky Survey) magnitudes (Borucki et al. 2011; Batalha et al. 2012). Kepler's gas giant planets are shown as circles and rocky planets as squares. See the electronic edition of the PASP for a color version of this figure. 
offer a greatly improved observing cadence, resulting in a more complete survey and better-characterized transit curves.

Due to the fundamental selection biases of transit surveys of modest duration, most transiting planets will be found in shortperiod orbits of a few months or less, but only for late-type stars does this preferred orbital location coincide with the habitable zone. A near-IR survey ( $\$ 1$, Fig. 1) would find 3-7 times more rocky planets orbiting $\mathrm{K}$ and $\mathrm{M}$ stars than would an otherwiseidentical visible-light survey-a critical difference for the identification of planets with temperate atmospheres $(250 \mathrm{~K}<$ $T_{\text {eff }}<350 \mathrm{~K}$ ), which are of great interest for spectroscopic follow-up. The laboratory experiments described in this article demonstrate that the performance of modern near-IR detectors is adequate for such a survey and for subsequent follow-up observations.

\subsection{Future Work}

Experiments with the existing dewar configuration are currently underway to correlate spatial variations detector performance (e.g., dark current, responsivity, and linearity) with photometric performance, both to identify whether calibrations can improve upon the photometric performance obtained through PCA alone and to establish criteria for excluding "bad pixels." These criteria will assist in developing metrics for detector screening and grading for possible future missions. Future experiments in this series will include (1) an examination of the effect on photometric precision of varying the number of pixels within the stellar image that is directly relevant to JWST/ NIRCam observations using weak lenses to defocus NIRCam images and (2) simulations of the extraction of transit spectra over a wide range of spectral line properties: e.g., line depth and line width. These results will be relevant to photometric, grism, and spectroscopic modes of JWST, as well as to dedicated transit spectroscopy missions presently under consideration in the United States and Europe.

The research described in this article was carried out in part at the Jet Propulsion Laboratory (JPL), California Institute of Technology, under a contract with the National Aeronautics and Space Administration. Support from the JPL Research and Technology Development program and from the Near-IR Camera (NIRCam) instrument on James Webb Space Telescope is gratefully acknowledged. We are grateful to Thomas Greene and Jim Beletic for useful discussions. The HR2G detector used for these experiments was provided by the Lawrence Berkeley National Laboratory. We used NASA's Exoplanet Archive in the preparation of this article.

\section{REFERENCES}

Baglin, A., Auvergne, M., Barge, P., et al. 2009, in IAU Symp. 253, Transiting Planets (Cambridge: Cambridge Univ. Press), 71

Bakos, G. Á., Noyes, R. W., Kovács, G., et al. 2009, in IAU Symp. 253, Transiting Planets (Cambridge: Cambridge Univ. Press), 21

Barman, T. 2007, ApJ, 661, L191

Barman, T. S. 2008, ApJ, 676, L61

Barron, N., Borysow, M., Beyerlein, K., et al. 2007, PASP, 119, 466

Basri, G., Walkowicz, L. M., Batalha, N., et al. 2010, ApJ, 713, L155

Batalha, N., et al. 2012, ApJS, in press

Bean, J. L., Seifahrt, A., Hartman, H., et al. 2010, ApJ, 713, 410

Beaulieu, J.-P., Tinetti, G., Kipping, D. M., et al. 2011, ApJ, 731, 16

Beichman, C. A., Greene, T., \& Krist, J. 2009, IAU Symp. 253,

Transiting Planets (Cambridge: Cambridge Univ. Press), 319

Beletic, J. W., Blank, R., Gulbransen, D., et al. 2008, Proc. SPIE, 7021, $70210 \mathrm{H}$

Berta, Z. K., Charbonneau, D., Désert, J.-M., et al. 2012, ApJ, 747, 35

Biesiadzinski, T., Lorenzon, W., Newman, R., et al. 2011, PASP, 123, 958

Borucki, W. J., \& Koch, D. G. 2011, in IAU Symp. 276, The Astrophysics of Planetary Systems: Formation, Structure, and Dynamical Evolution (Cambridge: Cambridge Univ. Press), 34

Borucki, W. J., Koch, D. G., Basri, G., et al. 2011, ApJ, 728, 117

Brown, M., Schubnell, M., \& Tarle, G. 2006, PASP, 118, 1443

Bryden, G., Stauffer, J., Ciardi, D. R., \& NStED Science Team 2011, BAAS, 43, 140.20

Charbonneau, D., Berta, Z. K., Irwin, J., et al. 2009, Nature, 462, 891

Ciardi, D. R., von Braun, K., Bryden, G., et al. 2011, AJ, 141, 108

Clampin, M. 2010, in ASP Conf. Ser. 430, Pathways Towards Habitable Planets (San Francisco: ASP), 167

Claudi, R. 2010, Ap\&SS, 328, 319
Deming, D., Harrington, J., Seager, S., \& Richardson, L. J. 2006, ApJ, 644,560

Deming, D., Seager, S., Winn, J., et al. 2009, PASP, 121, 952

Demory, B. O., Gillon, M., \& Seager, S., et al. 2012, ApJL, 751, L28

Désert, J.-M., Lecavelier des Etangs, A., Hébrard, G., et al. 2009, ApJ, 699, 478

Désert, J.-M., Sing, D., Vidal-Madjar, A., et al. 2011, A\&A, 526, A12

Finger, G., Mehrgan, H., Meyer, M., et al. 2000, Proc. SPIE, 4008, 1280

Frasca, A., Covino, E., Spezzi, L., et al. 2009, A\&A, 508, 1313

Gibson, N. P., Pont, F., \& Aigrain, S. 2011, MNRAS, 411, 2199

Gould, A., Pepper, J., \& DePoy, D. L. 2003, ApJ, 594, 533

Hardy, T., Baril, M. R., Pazder, J., \& Stilburn, J. S. 2008, Proc. SPIE, 7021, 70212B

Hauschildt, P. H., Allard, F., Ferguson, J., Baron, E., \& Alexander, D. R. 1999, ApJ, 525, 871

Howard, A. W., Marcy, G. W., Bryson, S. T., et al. 2011, ApJ, in press (arXiv:1103.2541)

Howe, D. A., Allan, D. W., \& Barnes, J. A. 1981, in Proc. 35th Annual Frequency Control Symp. (Piscataway: IEEE), A1

Irwin, J., et al. 2010, ApJ, 718, 1353

Jenkins, J. M., Dunham, E. W., Argabright, V. S., et al. 2011, in ESS Meeting 2 (New York: AAS), 1914

Jenkins, J. S., Murgas, F., Rojo, P., et al. 2011, A\&A, 531, A8

Johnson, J. A., Winn, J. N., Cabrera, N. E., \& Carter, J. A. 2009, ApJ, 692, L100

Knutson, H. A., Charbonneau, D., Allen, L. E., et al. 2007, Nature, 447, 18

Knutson, H. A., Madhusudhan, N., Cowan, N. B., et al. 2011, ApJ, 735, 27 
NIR PHOTOMETRY FOR EXOPLANET TRANSITS. I. 713

Plavchan, P., \& Anglada, G. 2011, in ESS Meeting 2 (New York: AAS), 20.01

Pollacco, D. L., Skillen, I., Collier Cameron, A., et al. 2006, PASP, 118, 1407

Reiners, A., Bean, J. L., Huber, K. F., et al. 2010, ApJ, 710, 432

Ricker, G. R., Latham, D. W., Vanderspek, R. K., et al. 2010, BAAS, 42, 450.06

Smith, R. M., Zavodny, M., Rahmer, G., \& Bonati, M. 2008, Proc. SPIE, 7021, 70210K
Stevenson, K. B., Harrington, J., Nymeyer, S., et al. 2010, Nature, 464, 1161

Swain, M. R., Bouwman, J., Akeson, R. L., Lawler, S., \& Beichman, C. A. 2008, ApJ, 674, 482

Swain, M. R., Vasisht, G., Tinetti, G., et al. 2009, ApJ, 690, L114

Tessenyi, M., Ollivier, M., Tinetti, G., et al. 2012, ApJ, 746, 45

Tinetti, G., Vidal-Madjar, A., Liang, M.-C., et al. 2007, Nature, 448,169

Tinetti, G., et al. 2011, Exp. Astron., accepted (arXiv:1112.2728) 